\title{
Visuo-motor integration in unresponsive wakefulness syndrome: A piece of the puzzle towards consciousness detection?
}

\author{
Antonino Naro ${ }^{\mathrm{a}}$, Antonino Leo ${ }^{\mathrm{a}}$, Serena Filoni ${ }^{\mathrm{b}}$, Placido Bramanti ${ }^{\mathrm{a}}$ and Rocco Salvatore Calabrò ${ }^{\mathrm{a}, *}$ \\ a IRCCS Centro Neurolesi “Bonino-Pulejo” Messina, S.S, Contrada Casazza, Messina, Italy \\ ${ }^{\mathrm{b}}$ Fondazione Centri di Riabilitazione Padre Pio Onlus, Viale Cappuccini, San Giovanni Rotondo (FG), Italy
}

\begin{abstract}
.
Purpose: The unresponsive wakefulness syndrome (UWS) is characterized by either a profound unawareness or an impairment of large-scale cortico/subcortical connectivity. Nevertheless, some individuals with UWS could show residual markers of consciousness and cognition. In this study, we applied an electrophysiological approach aimed to identify the residual visuomotor connectivity patterns that are thought to be linked to awareness, in patients with chronic disorder of consciousness (DOC).

Methods: We measured some markers of visuomotor and premotor-motor integration in 14 patients affected by DOC, before and after the application of transcranial direct current stimulation, delivered over the dorsolateral prefrontal cortex and the parieto-occipital area, paired to transorbital alterning current stimulation.

Results: Our protocol induced a potentiation of the electrophysiological markers of visuomotor and premotor-motor connectivity, paired to a clinical improvement, in all of the patients with minimally conscious state and in one individual affected by UWS. Conclusions: Our protocol could be a promising approach to potentiate the functional connectivity within large-scale visuomotor networks, thus allowing identifying the patients suffering from a functional locked-in syndrome (i.e. individuals showing an extreme behavioral motor dysfunction although with somehow preserved cognitive functions that can be identified only through para-clinical tests) within individuals with UWS.
\end{abstract}

Keywords: DLPFC, functional connectivity, MCS, parieto-occipital area, tDCS, UWS, visuomotor integration

\section{Introduction}

The clinical detection of awareness signs, including purposeful behavioral responsiveness to stimuli, has a pivotal role in the differential diagnosis of chronic disorders of consciousness (DOC). In fact, the lack of awareness characterizes the unresponsive wakefulness syndrome (UWS) (previously named vegetative state - VS), whereas patients with mini-

${ }^{*}$ Corresponding author: Rocco Salvatore Calabrò, IRCCS Centro Neurolesi "Bonino-Pulejo" Messina, S.S. 113, Contrada Casazza, 98124, Messina, Italy. Tel.: +39 09060128954; Fax: +39 09060128950; E-mail: salbro77@ tiscali.it. mally conscious state (MCS) show "inconsistent but clearly discernible behavioral evidence of consciousness" (Giacino et al., 2002; Laureys et al., 2010a). Notably, awareness impairment and, thus, the limitation of behavioral responsiveness are proportionally related to the level of connectivity disruption within a wide cortical/subcortical neuromatrix that supports consciousness generation and maintenance, besides the arousal system(Laureys etal.,2010b). Nevertheless, the patients suffering from a functional locked-in syndrome (fLIS) show an extreme behavioral motor dysfunction although with a partial preservation of higher cognitive functions and cerebral connectivity, as advanced neurophysiological and neuroimaging approaches have 
shown (Bruno et al., 2011). Such condition may arise from lesions at one or more levels of the sensory-motor system. On the other hand, patients with classic (i.e., quadriplegia and anartria, with eye-coded communication) and total LIS (i.e., a rare syndrome characterized by complete immobility including eye movements) typically suffer from a brainstem injury and show very limited signs of awareness due to profound sensory and motor deficits, although with preserved self-awareness and cognitive capacities, besides a normal brain network connectivity (Haig et al., 1987; Laureys et al., 2005). Indeed, it is extremely difficult to properly assess awareness in such patients, since either their movements may be minimal or inconsistent, or no cognitive output could be possible (Giacino and Zasler, 1995). In fact, fLIS, total LIS, and patients suffering from UWS are behaviorally indistinguishable, and it is possible to reach the differential diagnosis only through paraclinical approaches, including functional neuroimaging and advanced neurophysiological paradigms (Bruno et al., 2011).

Hence, a covert cognition and a residual complex cerebral connectivity characterize patients affected by fLIS and total LIS, whereas individuals with UWS lack of such markers. Nevertheless, the study of a sensory-motor system that is unable to contribute to the generation of purposeful behaviors, is challenging in patients with DOC, and thus the misdiagnosis rate is still high (Bekinschtein and Manes, 2008).

To this end, the study of large-scale sensory-motor integration processes supporting awareness may be useful in an attempt to differentiate patients with fLIS (Kotchoubey et al., 2013). In particular, the visuomotor integration (VMI) assessment seems a promising approach, as suggested by its clinical usefulness in terms of differential diagnosis and prognosis (Troiano et al., 2012; Hildebrandt et al., 2007). Moreover, VMI enrolls different and complex cortical-subcortical networks that are related to visual processes at conscious level (Grossberg, 2003; Humphreys et al., 1997). On the other hand, the first-level VMI processes (i.e. the paired activation of primary motor and primary visual cortices) do not correlate with awareness preservation (reflecting instead alerting functions, motor attention and preparation) (Katsuki and Constantinidis, 2012; Monti et al., 2013).

Several works have shown the possibility to bring to light the above mentioned complex integrative processes by means of non-invasive neurostimulation techniques, including the repetitive transcranial mag- netic stimulation (rTMS) and the transcranial direct current stimulation (tDCS). Such approaches could boost neural plasticity by means of long-term potentiation or depression-like mechanisms (LTP or LTD) (Ziemann et al., 2008) within the primary motor cortex (M1) (Rioult-Pedotti et al., 2000; Sanesand Donoghue, 2000), the sensory-motor areas (Stefan et al., 2000; Wolters et al., 2003), and the pain-matrix (GarciaLarrea and Peyron, 2013; Suppa et al., 2013; Naro et al., 2015b). In addition, such paradigms do not necessarily require a substantial patient's cooperation. Interestingly, the assessment and the modulation of VMI through non-invasive neuromodulation could be helpful in the differential diagnosis of UWS and fLIS. Indeed, we may argue that patients with UWS who show an improvement of the cortical functional connectivity and the visuomotor output after a proper neuromodulation approach should be no longer considered as UWS, but fLIS. To this end, we assessed the large-scale visuo-premotor-motor functional connectivity in a clinically defined UWS sample and in a control group of MCS and healthy subjects (HC), before and after the administration of a paired associative stimulation protocol consisting of tDCS over dorsolateral prefrontal cortex (DLPFC) and parieto-occipital areas (POA), and trans-orbital alterning current stimulation (tACS). More in detail, we measured some parameters of cortical excitability and connectivity, by means of single- and dual-site TMS, and the visual-stimuli event-related potentials. Since a standard visual pathway stimulation is extremely challenging in patients with DOC, owing to the low and inconsistent cooperation, we chose a tACS approach capable to evoke visual potentials regardless patient's cooperation. We stimulated the DLPFC and the POA by means of the dual-site tDCS, because of their important role in the stimulus-driven (bottom-up) and the purpose-driven (top-down) neural processes (Grossberg, 2003; Humphreys et al., 1997). More in detail, the POA's intrinsic circuits constitute the common origin of three distinct pathways: i) the parieto-prefrontal, involved in top-down control of eye movements and in spatial working memory; ii) the parieto-premotor, mediating ocular, reaching, and grasping movements; and iii) the parieto-temporal, underlying the complex spatial processing required for navigating through the environment.

Since voluntary eye-movements are an important clinical marker of the level of awareness, we chose such double-site tDCS approach. 
Table 1

The Clinical and demographic characteristics of the whole sample. We reported the individual CRS-R mean values \pm sd (the CRS-R was daily administered for 30 consecutive days before the protocol enrollment). We marked in bold the patients who showed a visuo-motor improvement after the real_protocol application

\begin{tabular}{|c|c|c|c|c|c|c|c|c|c|c|c|c|}
\hline \multirow[t]{2}{*}{$\overline{\mathrm{DOC}}$} & \multirow[t]{2}{*}{ gender } & \multirow[t]{2}{*}{ etiology } & \multirow[t]{2}{*}{ age } & \multirow[t]{2}{*}{$\mathrm{BI}$} & \multirow[t]{2}{*}{ MRI } & \multicolumn{7}{|c|}{ CRS-R } \\
\hline & & & & & & total & A & V & M & OM & $\mathrm{C}$ & $\mathrm{Ar}$ \\
\hline \multirow[t]{7}{*}{$\overline{\mathrm{MCS}}$} & $\mathrm{F}$ & A & 72 & 6 & WMH & $18 \pm 0.7$ & $4 \pm 1.4$ & $4 \pm 0.6$ & $5 \pm 1.9$ & $1 \pm 1.8$ & $1.8 \pm 0.9$ & $3 \pm 1.3$ \\
\hline & M & $\mathrm{T}$ & 51 & 18 & WMH, ${ }^{R}$ BG_h & $15 \pm 1.6$ & $3 \pm 1.5$ & $3 \pm 0.9$ & $4 \pm 1.7$ & $1 \pm 1.2$ & $0.6 \pm 0.3$ & $3 \pm 0.8$ \\
\hline & $\mathrm{F}$ & A & 66 & 9 & WMH & $12 \pm 1.7$ & $1 \pm 0.9$ & $3 \pm 1.2$ & $2 \pm 0.8$ & $2 \pm 0.7$ & $1.6 \pm 0.9$ & $3 \pm 0.9$ \\
\hline & $\mathbf{F}$ & $\mathbf{T}$ & 70 & 22 & ${ }^{\mathrm{L}} \mathbf{F b} \_\mathbf{h}$ & $15 \pm 0.6$ & $3 \pm 1.6$ & $2 \pm 0.3$ & $5 \pm 0.5$ & $\mathbf{1} \pm \mathbf{0 . 6}$ & $0.6 \pm 0.2$ & $\mathbf{3} \pm \mathbf{1 . 9}$ \\
\hline & M & $\mathbf{T}$ & 33 & 8 & multiple h & $\mathbf{1 3} \pm \mathbf{0 . 8}$ & $2 \pm 1$ & $2 \pm 0.7$ & $3 \pm \mathbf{0 . 5}$ & $2 \pm 1.2$ & $1.9 \pm 0.5$ & $\mathbf{3} \pm \mathbf{1 . 4}$ \\
\hline & $\mathrm{F}$ & A & 41 & 15 & WMH & $12 \pm 1$ & $1 \pm 0.5$ & $1 \pm 0.6$ & $3 \pm 0.5$ & $2 \pm 0.3$ & $0.6 \pm 0.4$ & $3 \pm 1.9$ \\
\hline & M & $\mathrm{T}$ & 35 & 16 & WMH, ${ }^{R}$ BG_h & $11 \pm 0.2$ & $1 \pm 1.8$ & $1 \pm 1.7$ & $3 \pm 0.7$ & $2 \pm 1.8$ & $1.7 \pm 0.6$ & $3 \pm 0.1$ \\
\hline mean $\pm s d$ & & & $53 \pm 17$ & $13 \pm 6$ & & $14 \pm 0.9$ & $2 \pm 1.2$ & $2 \pm 0.9$ & $4 \pm 0.9$ & $1.6 \pm 1.1$ & $1 \pm 1.3$ & $3 \pm 1.1$ \\
\hline \multirow[t]{7}{*}{ UWS } & M & A & 53 & 8 & WMH & $5 \pm 0.4$ & $1 \pm 1.1$ & $1 \pm 1.2$ & $1 \pm 1.1$ & $1 \pm 0.6$ & $0.4 \pm 0.1$ & $1 \pm 1.5$ \\
\hline & $\mathrm{F}$ & $\mathrm{T}$ & 26 & 3 & DAI, SAH & $4 \pm 1.4$ & $1 \pm 0.7$ & $1 \pm 0.8$ & $1 \pm 1.5$ & $0 \pm 1.4$ & $1.3 \pm 1$ & $1 \pm 0.6$ \\
\hline & $\mathbf{F}$ & $\mathbf{T}$ & 66 & 8 & ${ }^{\mathrm{R}} \mathbf{F P} \_\mathbf{h}$ & $7 \pm 1$ & $\mathbf{0} \pm \mathbf{1 . 2}$ & $2 \pm 1.5$ & $2 \pm 1.4$ & $1 \pm 1.4$ & $\mathbf{1} \pm \mathbf{1}$ & $\mathbf{1} \pm \mathbf{2}$ \\
\hline & $\mathrm{F}$ & A & 62 & 11 & WMH & $6 \pm 1$ & $1 \pm 1.3$ & $1 \pm 1.2$ & $2 \pm 0.6$ & $0 \pm 0.3$ & $0.8 \pm 0.9$ & $2 \pm 0.4$ \\
\hline & M & $\mathrm{T}$ & 61 & 9 & $\mathrm{SAH}$ & $4 \pm 0.7$ & $1 \pm 1$ & $1 \pm 0.2$ & $1 \pm 0.2$ & $0 \pm 1.2$ & $1.1 \pm 0.2$ & $1 \pm 1.6$ \\
\hline & M & A & 69 & 11 & WMH & $7 \pm 1.3$ & $1 \pm 0.1$ & $1 \pm 0.7$ & $2 \pm 0.7$ & $1 \pm 1.1$ & $1.4 \pm 0.6$ & $2 \pm 0.9$ \\
\hline & $\mathrm{F}$ & $\mathrm{T}$ & 74 & 12 & DAI, SAH & $6 \pm 0.3$ & $1 \pm 1.4$ & $2 \pm 1.4$ & $1 \pm 1.4$ & $0 \pm 0.7$ & $0.4 \pm 0.6$ & $2 \pm 1.4$ \\
\hline mean $\pm s d$ & & & $59 \pm 16$ & $9 \pm 3$ & & $6 \pm 1$ & $0.9 \pm 0.4$ & $1.3 \pm 0.5$ & $1.5 \pm 0.5$ & $0.4 \pm 0.5$ & $0.9 \pm 0.6$ & $1.6 \pm 0.5$ \\
\hline
\end{tabular}

Etiology: A, post-anoxic, T, post-traumatic brain injury; BI: brain injury onset in months; age in years; MRI: structural patterns including WMH (white matter hyper-intensity), h h (hemorrhagic lesion), ${ }^{\mathrm{R}} \mathrm{FP}$ (right fronto-polar), ${ }^{\mathrm{R}} \mathrm{BG}$ (basal ganglia), ${ }^{\mathrm{L}} \mathrm{Fb}$ (left fronto-basal), SAH (sub-arachnoid hemorrhage), DAI (diffuse axonal injury); CRS-R: Coma Recovery Scale-revised including auditory (A), visual (V), motor (M), oro-motor (OM), communication domain (C), and arousal induction (Ar); sd: standard deviation.

We hypothesized that our experimental protocol could improve the motor area excitability, the visuoprefrontal functional connectivity, and the visuomotor output, allowing bringing to light covert signs of awareness in patients who were clinically defined UWS.

\section{Methods}

\subsection{Subjects}

Of the 32 subjects suffering from chronic DOC attending the Neurorehabilitative Unit of the IRCCS Centro Neurolesi "Bonino-Pulejo" (Messina, Italy), we enrolled 14 patients who met the criteria for VS/MCS diagnosis (The Multi-Society Task Force on PVS, 1994; Giacino et al., 2002), and the following exclusion criteria: a DOC condition lasting less than 3 months after the brain injury; other severe neurological or systemic diseases; critical conditions (i.e. inability to breathe independently, hemodynamic instability); cortical excitability-modifying drugs assumption beyond L-DOPA and baclofen; presence of epileptic history, pace-maker, aneurysms clips, neurostimulator, brain/subdural electrodes or other electromechanical devices; presence of electroencephalographic (EEG) suppression-burst pattern; absence of visual evoked potential (VEP). In addition, we included in the study $7 \mathrm{HC}$ (4 females and 3 males, mean age $55.3 \pm 5.8$ years) as control group.

We reported the clinic-demographic characteristics in Table 1. DOC etiology consisted of a post-anoxic or a post-traumatic brain damage. The neurological examination mainly showed a pattern of spastic tetraparesis. EEG examination evidenced a continuous slowing in theta and/or delta frequency ranges. Our Research Institute Ethics Committee approved the present study and either the $\mathrm{HC}$ or the legal guardian of each patient gave their written informed consent.

\subsection{Clinical assessment}

Two neurologists skilled in DOC diagnosis independently evaluated the patients through the JFK CRS-R. This scale is a reliable and standardized tool, which integrates neuropsychological and clinical assessment, and includes the current diagnostic criteria for coma, VS, and MCS, allowing the clinician to assign the patient to the most appropriate diagnostic category. Hence, the CRS-R represents an appropriate approach for characterizing the level of consciousness and 
for monitoring the neurobehavioral function recovery (Gerrard et al., 2014). The CRS-R was daily administered for 30 consecutive days, at different times, in order to steadily establish the level of consciousness impairment.

\subsection{Conditioning protocols}

Each participant underwent four different protocols: i) a real (real_tDCS+real_tACS); ii) a sham (sham tDCS+sham_tACS); iii) a tDCS_alone (real_tDCS + sham_tACS); and iv) a tACS_alone (real_tACS+ sham_tDCS). We administered the protocols in a random scheme (i, ii, iii, and iv) and in different sessions, at one-day of interval. Participants and experimenters who analyzed data were blinded on scheme procedure.

For the real_tDCS, we used a battery-driven stimulator (Brain Stim, E.M.S., Bologna, Italy) with a couple of conductive-rubber electrodes, placed in saline-soaked sponges (active electrode $5 \times 5 \mathrm{~cm}$ ) over F3 (DLPFC) and PO5 (POA) (according to the 10/20 International System). The current stimulation ramped up/down during the first/last $30 \mathrm{sec}$ of stimulation, at $1 \mathrm{~mA}$ of intensity. Current density was always below the safety limit of $52 \mu \mathrm{A} / \mathrm{cm}^{2}$. The device kept the impedance below $10 \mathrm{k} \Omega$ (Miranda et al., 2006; Bikson et al., 2010; Nitsche et al., 2003).

For the real_tACS, we extra-ocularly applied 4 electrodes (sintered $\mathrm{Ag} / \mathrm{AgCl}$ ring electrodes) to both eyelids of the left eye (while the eye was kept closed), cabled to a battery-driven stimulator (BrainStim, E.M.S., Bologna, Italy) (Sabel et al., 2011). FPz served as reference electrode. At first, we determined the phosphene threshold in our HC sample, applying bursts of 15 pulses $(10 \pm 2 \mathrm{~ms}$ at $20 \pm 5 \mathrm{~Hz}$; the values ranged randomly in order to avoid habituation phenomena) and increasing the current intensity by steps of $10 \mu \mathrm{A}$ per second (starting from zero). We set the stimulation frequency in $\mathrm{HC}$, increasing the frequency by steps of $1-5 \mathrm{~Hz}$ per second, from the $\alpha$-range (the minimum stimulation frequency applicable) to the flicker-fusion frequency (i.e. the maximal temporal resolution frequency at which the intermittent tACS stimulus appeared as steady to the average of the $\mathrm{HC}$ ), at an intensity of $\leq 0.8 \mathrm{~mA}$. The mean values of these parameters were applied in each participant affected by DOC.

Concerning the real_protocol, we delivered 150 visual stimuli during each tDCS protocol, at the same intensity used in VEP elicitation, at $0.25 \mathrm{~Hz}$ (i.e., 300 stimuli in $20 \mathrm{~min}$ ). For the sham_tDCS, we switched- off the stimulator after $30 \mathrm{sec}$, whereas the sham_tACS consisted of 50 stimuli (i.e. at $0.08 \mathrm{~Hz}$ ).

\subsection{Single-pulse, paired-pulse and dual-site TMS measures}

We used a high-power Magstim200² Stimulator (Magstim, Whitland, Dyfed, UK) in order to elicit the motor evoked potential (MEP) through magnetic monophasic stimuli. We held the coil tangentially to the scalp with the handle pointing backwards and laterally, at a $45^{\circ}$ angle from the sagittal plane, approximately perpendicular to the central sulcus of the left hemisphere, on the optimal scalp site to get the wider MEP amplitude from the relaxed right abductor pollicis brevis muscle (APB) (motor hot-spot). The rise time of the magnetic monophasic stimulus was $\sim 100 \mu$ s with a to-zero of $\sim 800 \mu \mathrm{s}$. The current flowed in handle direction during the rise-time of the magnetic field, thus with a posterior-anterior direction. We preliminarily evaluated the resting motor threshold (RMT), defined as the smallest magnetic stimulus intensity able to evoke a peak-to-peak MEP of $50 \mathrm{uV}$ in resting right $\mathrm{APB}$, in at least five-out-ten consecutive tracks (Rossini et al., 1994). Then, we applied an intensity of stimulation of $120 \%$ of RMT (test stimulus). We applied $\mathrm{Ag}-\mathrm{AgCl}$ surface electrodes to the right $\mathrm{APB}$, using a classic muscle belly-tendon montage, for recording EMG activity. A Digitimer D360 Amplifier (Digitimer Ltd., Welwyn Garden City, Herts, UK) amplified, filtered $(32 \mathrm{~Hz}-1 \mathrm{KHz})$, and stored the signals (at $10 \mathrm{KHz}$ on a personal computer for off-line analysis) (Signal Software, Cambridge Electronic Design, Cambridge, UK). During the experiments, we continuously monitored the EMG activity through visual (oscilloscope) and auditory (speakers) feedback, to ensure the complete muscle relaxation.

Concerning the short-latency intracortical facilitation (SICF), we delivered two juxta-RMT stimuli (being the former the conditioning stimulus, the latter the test one) at an interstimulus interval of $2.5 \mathrm{~ms}$ (Tokimura et al., 1996), whereas we applied two suprathreshold stimuli (at 120\% RMT) at an interstimulus interval of $150 \mathrm{~ms}$ for the long-latency intracortical inhibition (LICI) (Valls-Sole et al., 1992). We measured the mean amplitude of the conditioned MEP as percentage of the amplitude of the unconditioned MEP (test), which was taken as a measure of cortical excitability. We registered 15 unconditioned MEPs, 10 LICI, and 10 SICF, randomly intermingled in a single 
trial, at a frequency of $0.25 \mathrm{~Hz}$ (test duration $\sim 9 \mathrm{~min}$ ). All data are given as mean or percentage difference in comparison to baseline values \pm standard error (se).

Concerning premotor-motor interaction, we measured the effects on MEP amplitude of a dual-site TMS approach (using two figure-of-eight monophasic coils) on $\mathrm{PMd}_{\text {right }}-\mathrm{M} 1_{\text {left }}$ and $\mathrm{PMv} v_{\text {left }}-\mathrm{M} 1_{\text {left }}$ (interstimulus interval of $7 \mathrm{~ms}$, conditioning stimulus at $70 \%$ RMT on the premotor area, test stimulus over M1 at $120 \%$ RMT), SMA-M1 left (CS at an interstimulus interval of $6 \mathrm{~ms}$, conditioning stimulus at $3 \mathrm{~cm}$ anteriorly to $\mathrm{Cz}$-preSMA- at $70 \% \mathrm{RMT}$, test stimulus over M1 at $120 \% \mathrm{RMT}$ ), and $\mathrm{PPC}_{\text {left }}-\mathrm{M} 1_{\text {left }}$ (conditioning stimulus at an interstimulus interval of $10 \mathrm{~ms}$, at $90 \%$ RMT, test stimulus over M1 at $120 \%$ RMT) (Civardi et al., 2001; Koch et al., 2007a,b; Davare et al., 2008). We registered 10 conditioned MEPs for each premotor-motor interaction in different trials, intermingled with 10 unconditioned MEPs, delivered at a frequency of $0.25 \mathrm{~Hz}$ (test duration $\sim 12 \mathrm{~min}$ ). We measured the mean amplitude of the conditioned MEP as percentage of the amplitude of the unconditioned (test) MEP, which was taken as a measure of premotor-motor excitability. All data are given as mean or percentage difference in comparison to baseline values \pm se.

\subsection{VEP, VMI, and ERP}

Since a standard VEP assessment in patients with DOC is extremely challenging owing to the low and inconsistent cooperation, we chose a tACS approach in order to elicit VEP (Gall et al., 2010, 2011). We delivered 100 visual stimuli over left eye, while kept closed, with the same characteristics used for tACS, at a frequency of $1 \mathrm{~Hz}$ (test duration $\sim 1.6 \mathrm{~min}$ ). We recorded the EEG activity from 3 scalp sites, using $\mathrm{Ag} / \mathrm{AgCl}$ electrodes positioned over the mid-occipital (MO, $5 \mathrm{~cm}$ above nasion), left lateral occipital (LLO, $5 \mathrm{~cm}$ lateral to MO), and left temporal area (LT, $5 \mathrm{~cm}$ lateral to LLO) (Suppa et al., 2015). An electrode over the mid-frontal position (12 cm above nasion) served as reference, whereas one over right mastoid as ground. Impedance was $\leq 5 \mathrm{k} \Omega$. We amplified and acquired the signals at $5 \mathrm{kHz}$ through a 1401plus AD laboratory interface (Cambridge Electronic Design, Cambridge, UK), registered and filtered $(0.5-70 \mathrm{~Hz}+50 \mathrm{~Hz}$ notch) through a Digitimer D360 (Digitimer Ltd, Welwyn Garden City, UK), and stored on a personal computer for off-line analysis (Signal software; Cambridge Electronic Design, UK). We registered a biphasic evoked component (N75-P100) from MO in HC, which was similar to standard pattern-reversal VEP.

In a different VEP session, we applied a tACS protocol in analogy to an odd-ball paradigm (Machado et al., 2014), in which we randomly shifted the stimulation frequency and the intensity of $\pm 25 \%$ in an 80:20 frequent:infrequent ratio. Each participant underwent a block of 500 trials (test duration $\sim 8 \mathrm{~min}$ ), in which there was a $95 \%$ chance of 1-4 infrequent stimuli preceding a frequent one and a 5\% chance of 5-7 infrequent preceding a frequent one. Hence, we were able to register an ERP from LLO and LT regardless participant's cooperation. For ERP analysis, we filtered at $0.3 / 25 \mathrm{~Hz}+$ notch $(50 \mathrm{~Hz})$, epoched (ranging from $-100 \mathrm{~ms}$ by visual stimulus onset to $600 \mathrm{~ms}$ after) after visual and independent component analysis inspection for artifact removal, and averaged EEG signals for each channel. Then, we measured the amplitude of the registered ERP.

We assessed the VMI by means of paired visual stimuli (conditioning stimulus through left-eye tACS) and MEP (test stimulus by means of single-pulse TMS over $\mathrm{M} 1_{\text {left }}$ ). We chose two individually-adapted interstimulus intervals that were clearly linked to inhibitory (VEP latency $+40 \mathrm{~ms}$ ) or facilitatory (VEP latency $+100 \mathrm{~ms}$ ) effects on MEP amplitude in healthy individuals (Suppa et al., 2015). We registered 15 unconditioned MEP intermingled with 15 VEP-MEP interactions for each interstimulus intervals in a single trial, delivered at a frequency of $0.2 \mathrm{~Hz}$ (test duration $\sim 9$ min). We measured the mean amplitude of the conditioned MEP as percentage of the amplitude of the unconditioned (test) MEP, which was taken as a measure of VMI strength. All the data are given as mean or percentage difference in comparison to baseline values \pm se.

\subsection{Study design}

HC sat in a comfortable armchair in a darkened room, whereas we carried-out the patients' experimental procedure at bedside, in a darkened environment. $\mathrm{HC}$ and patients had their right eye covered by an eyepatch. We applied a real electrophysiological protocol, a sham, a tDCS_alone, and a tACS_alone protocol as control experiments, in each group. The protocols were delivered at one-day of interval, in a random delivery scheme (i, ii, iii, and iv). Before ( $\left.\mathrm{T}_{\mathrm{PRE}}\right)$ and after the end of each conditioning protocol (immediately, $\mathrm{T}_{0}, 30-\mathrm{min}, \mathrm{T}_{30}$, and 60-min, $\mathrm{T}_{60}$ ), we assessed 

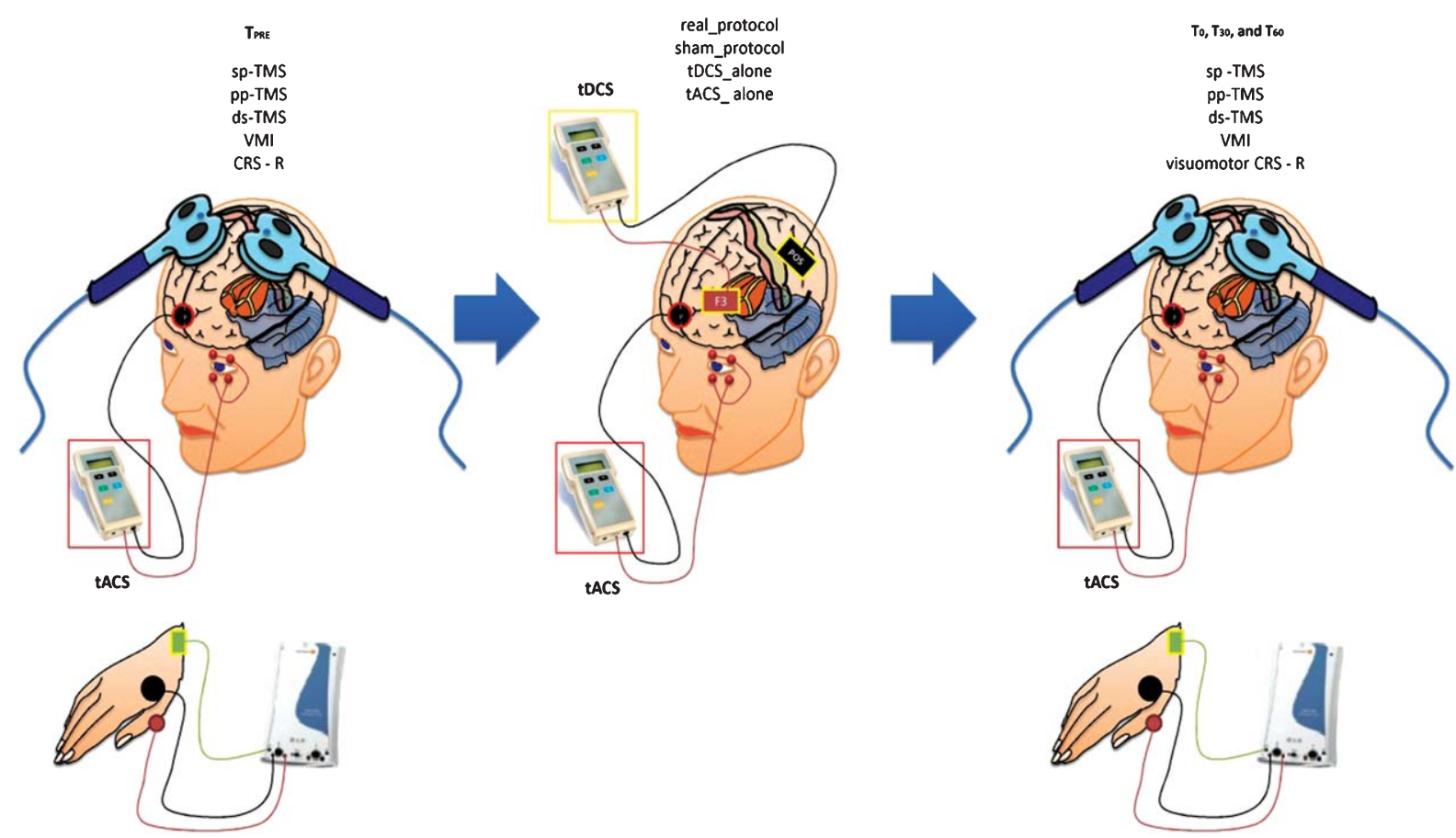

Fig. 1. Summarizes the experimental design. We carried the clinical (CRS-R) and the electrophysiological measurements (single pulse -sp-, paired-pulse -pp-, dual-site -ds- TMS, and visuomotor integration -VMI) before $\left(\mathrm{T}_{\mathrm{PRE}}\right)$ and after $\left(\mathrm{T}_{0}, \mathrm{~T}_{30}\right.$, and $\left.\mathrm{T}_{60}\right)$ each conditioning protocol: real_protocol, sham_protocol, tDCS_alone, and tACS_alone.

the peak-to-peak MEP amplitude from the left M1, the intracortical circuit excitability assessed through paired-pulse TMS over the left M1 (SICF and LICI), the premotor-motor interactions (PPC-M1, SMA-M1, PMd-M1, PMv-M1), and the VMI (combining tACS and TMS pulses over M1 at specific interstimulus intervals). Moreover, we measured the VEP and ERP parameters elicited by tACS. We individually adapted each site of TMS/tDCS stimulation and recording according to a recently performed brain MRI of each participant. We assessed the clinical effects of our protocols in patients with DOC through the JFK CRS-R. Figure 1 summarizes the experimental design.

\subsection{Data acquisition and statistical analysis}

We performed the baseline clinical and electrophysiological comparisons through unpaired $t$-tests. We evaluated the effects of the conditioning protocols on each dependent variable (visuomotor CRS-R, RMT, MEP, LICI, SICF, PMd-M1, PMv-M1, SMAM1, PPC-M1, VMI $40 \mathrm{~ms}, \mathrm{VMI}_{100 \mathrm{~ms}}, \mathrm{VEP}, \mathrm{ERP}_{\mathrm{LT}}$, and $\left.E_{R P} P_{L L O}\right)$ in separated three-way repeated-measure analyses of variance (rmANOVAs), implying time (four levels: $\mathrm{T}_{\mathrm{PRE}}, \mathrm{T}_{0}, \mathrm{~T}_{30}$, and $\mathrm{T}_{60}$ ) and protocol (four levels: real_protocol, sham_protocol, tDCS_alone, and tACS_alone) as within-subject factors, and group (three levels: MCS, UWS, and HC) as between-subject factor. The Greenhouse-Geisser method was used if necessary to correct for non-sphericity. Conditional on a significant F-value, we performed post-hoc t-tests (Bonferroni) to explore the strength of main effects and the patterns of interaction between the experimental factors. All statistical tests were applied two-tailed. A significant $p$-value was $<0.05$. All data are given as means or percent changes \pm se. We calculated a Fisher Z-transformation in order to assess an eventual correlation among clinical, demographic, and electrophysiological parameters.

\section{Results}

We did not observe any effects in patients and $\mathrm{HC}$, either during or after the entire experimental procedure. 
Table 2

The comparison among electrophysiological parameters in HC, MCS and individuals with UWS. We found significant differences between MCS and UWS (pMCS/UWS), and DOC and HC $\left(^{*}\right.$ ) (each unpaired $t$-tests, $p<0.05$, except for RMT\%, MEP amplitude, VEP amplitude and latency). UWS patients did not show ERPs, with a global impairment of cortical excitability, connectivity, and VEP latency and amplitude. MCS displayed an increase of cortical excitability (increased $\mathrm{VMI}_{100}$, SICF, PMv, and PPC; reduced VMI 40 , PMd, SMA, and LICI), a partially preserved premotor-motor and VMI connectivity, and the presence of ERPs limited to LO electrode. Some MCS/UWS differences approached the statistical significance (labeled as NS ${ }^{\text {unpaired }}$-test $)$. Data are reported as mean $\pm \mathrm{se}$

\begin{tabular}{|c|c|c|c|c|c|}
\hline & Measures & $\mathrm{HC}$ & MCS & UWS & $\mathrm{P}_{\mathrm{MCS} / \mathrm{UWS}}$ \\
\hline \multirow[t]{2}{*}{$\mathrm{sp}$} & RMT $(\%)$ & $55 \pm 3$ & $58 \pm 2$ & $60 \pm 9$ & NS \\
\hline & $\operatorname{MEP}(\mathrm{mV})$ & $0.7 \pm 0.1$ & $0.5 \pm 0.1$ & $0.4 \pm 0.1$ & NS \\
\hline \multirow[t]{4}{*}{ ds } & PMv-M1 (\%) & $125 \pm 9^{*}$ & $142 \pm 6$ & $105 \pm 7$ & $<0.001$ \\
\hline & PMd-M1 (\%) & $51 \pm 7^{*}$ & $92 \pm 7$ & $105 \pm 9$ & $\mathrm{NS}^{0.06}$ \\
\hline & SMA $(\%)$ & $53 \pm 9^{*}$ & $94 \pm 6$ & $106 \pm 8$ & $\mathrm{NS}^{0.07}$ \\
\hline & PPC-M1 (\%) & $123 \pm 11^{*}$ & $148 \pm 6$ & $105 \pm 7$ & $<0.001$ \\
\hline \multirow[t]{4}{*}{$\mathrm{pp}$} & $\operatorname{LICI}(\%)$ & $60 \pm 7^{*}$ & $94 \pm 13$ & $107 \pm 5$ & NS \\
\hline & $\operatorname{SICF}(\%)$ & $129 \pm 9^{*}$ & $144 \pm 13$ & $167 \pm 5$ & $\mathrm{NS}^{0.06}$ \\
\hline & $\mathrm{VMI}_{40}(\%)$ & $51 \pm 8^{*}$ & $79 \pm 6$ & $97 \pm 9$ & $<0.001$ \\
\hline & $\mathrm{VMI}_{100}(\%)$ & $112 \pm 5^{*}$ & $122 \pm 6$ & $108 \pm 9$ & 0.01 \\
\hline \multirow[t]{6}{*}{ tACS } & VEP latency (ms) & $102 \pm 7$ & $115 \pm 2$ & $121 \pm 3$ & NS \\
\hline & VEP amplitude $(\mu \mathrm{V})$ & $19 \pm 3$ & $12 \pm 2$ & $8 \pm 1$ & NS \\
\hline & $\mathrm{ERP}_{\text {LLO latency }}(\mathrm{ms})$ & $303 \pm 10^{*}$ & $340 \pm 15$ & Absent & $<0.001$ \\
\hline & $\mathrm{ERP}_{\text {LLO }}$ amplitude $(\mu \mathrm{V})$ & $17 \pm 8^{*}$ & $6 \pm 4$ & Absent & 0.002 \\
\hline & $\mathrm{ERP}_{\mathrm{LT}}$ latency $(\mathrm{ms})$ & $331 \pm 12^{*}$ & Absent & Absent & NS \\
\hline & $\mathrm{ERP}_{\mathrm{LT}}$ amplitude $(\mu \mathrm{V})$ & $10 \pm 4^{*}$ & Absent & Absent & NS \\
\hline
\end{tabular}

sp: single pulse TMS; ds: dual-site TMS; pp: paired-pulse TMS.

\section{Table 3}

We observed significant real_tDCS after-effects in HC and MCS patients, whereas UWS did not show any significant after-effect at group level. The tDCS_alone induced after-effects that were similar to the real_protocol but non-significant and limited to the MEP amplitude increase and the PPC-M1 potentiation. The tACS_alone and the sham_protocol were totally ineffective

\begin{tabular}{|c|c|c|c|c|c|}
\hline & \multirow{2}{*}{$\begin{array}{l}\text { timeXgroupXprotocol } \\
\text { interaction } \mathrm{F}_{(18,324)}, p\end{array}$} & & \multicolumn{3}{|c|}{ real_protocol } \\
\hline & & & $\mathrm{HC}_{(1,6)}, p$ & & $\operatorname{MCS} \mathrm{t}_{(1,6)}, p$ \\
\hline \multirow[t]{3}{*}{ MEP amplitude } & $3.0<0.001$ & $\mathrm{~T}_{0}$ & $3.2,0.02$ & & $3,0.01$ \\
\hline & & $\mathrm{T}_{30}$ & $2.7,0.03$ & & NS \\
\hline & & $\mathrm{T}_{60}$ & & NS & \\
\hline \multirow[t]{3}{*}{$\mathrm{ERP}_{\text {LLO }}$ amplitude } & $3.2,<0.001$ & $\mathrm{~T}_{0}$ & $6.1,<0.001$ & & $2.5,0.04$ \\
\hline & & $\mathrm{T}_{30}$ & $6,<0.001$ & & NS \\
\hline & & $\mathrm{T}_{60}$ & & NS & \\
\hline \multirow[t]{3}{*}{$\mathrm{ERP}_{\mathrm{LT}}$ amplitude } & NS & $\mathrm{T}_{0}$ & $6,<0.001$ & & NS \\
\hline & & $\mathrm{T}_{30}$ & $5.1,0.005$ & & NS \\
\hline & & $\mathrm{T}_{60}$ & & NS & \\
\hline \multirow[t]{3}{*}{ PMd-M1 \% } & $1.7,0.04$ & $\mathrm{~T}_{0}$ & $6.4,<0.001$ & & $2.3,0.04$ \\
\hline & & $\mathrm{T}_{30}$ & $3.2,0.02$ & & NS \\
\hline & & $\mathrm{T}_{60}$ & & NS & \\
\hline \multirow[t]{3}{*}{ PPC-M1 \% } & $3.8,<0.001$ & $\mathrm{~T}_{0}$ & $3.2,0.02$ & & $2.3,0.04$ \\
\hline & & $\mathrm{T}_{30}$ & $2.4,0.04$ & & NS \\
\hline & & $\mathrm{T}_{60}$ & & NS & \\
\hline \multirow[t]{3}{*}{ SICF \% } & $1.8,0.02$ & $\mathrm{~T}_{0}$ & $2.9,0.01$ & & $2.8,0.01$ \\
\hline & & $\mathrm{T}_{30}$ & $2.7,0.03$ & & NS \\
\hline & & $\mathrm{T}_{60}$ & & NS & \\
\hline \multirow[t]{3}{*}{$\mathrm{VMI}_{40} \%$} & $7.9,<0.001$ & $\mathrm{~T}_{0}$ & $9.1,<0.001$ & & $3.2,0.02$ \\
\hline & & $\mathrm{T}_{30}$ & $6.3,0.003$ & & NS \\
\hline & & $\mathrm{T}_{60}$ & & NS & \\
\hline \multirow[t]{3}{*}{$\mathrm{VMI}_{100} \%$} & $5.4,<0.001$ & $\mathrm{~T}_{0}$ & $3.8,0.003$ & & $3.2,0.02$ \\
\hline & & $\mathrm{T}_{30}$ & $3.2,0.02$ & & NS \\
\hline & & $\mathrm{T}_{60}$ & & NS & \\
\hline \multirow{3}{*}{ SMA-M1 \% } & $2.6,<0.001$ & $\mathrm{~T}_{0}$ & $2.8,0.01$ & & $2.8,0.01$ \\
\hline & & $\mathrm{T}_{30}$ & $2.7,0.03$ & & NS \\
\hline & & $\mathrm{T}_{60}$ & & NS & \\
\hline
\end{tabular}




\subsection{DOC/HC clinical and electrophysiological differences at baseline}

We showed the baseline CRS-R total scores in Table 1 (MCS-UWS comparison $p=0.003$ ). There were no differences concerning either DOC etiology or the demographic characteristics. We resumed in Table 2 the baseline DOC and HC electrophysiological differences. RMT, MEP amplitude, VEP amplitude and latency were similar in the three groups, whereas paired-pulse TMS, ERP, and VMI measures were significantly different concerning either DOC-HC $(p<0.05$ for each MCS$\mathrm{HC}$ and UWS-HC comparison, by means of unpaired $t$-tests) or MCS-UWS comparison (Table 2). Indeed, the patients suffering from DOC showed an increased facilitatory (SICF) and a strongly reduced inhibitory tone (SICI), a clear alteration of cortico-cortical interactions, with a prevalence of facilitatory connections, increased latency and reduced amplitudes of VEPs and visualERPs (although HC showed ERPs from both LLO and
LT electrodes, and the MCS only from LLO electrodes, whereas UWS did not show any ERP). Each parameter was more impaired in MCS than UWS.

\subsection{Conditioning protocol's effects on clinical assessment}

Although rmANOVA did not show any significant timeXgroupXprotocol interaction, we observed an increase of one point at the CRS-R visuomotor sub-item at $\mathrm{T}_{0}$ in the two patients with MCS (n. 4 and 5) and in one UWS (n. 3), only after the real_protocol. Indeed, such patients with MCS upgraded from "fixation" ( 2 points at the CRS-R visuomotor sub-item) to "following with eyes an object" (3 points), whereas the UWS from "visual startle" (1 point) to "visual fixation" (2 points). Such effects were short-lasting, since they were not detectable at either $\mathrm{T}_{30}$ or $\mathrm{T}_{60}$.
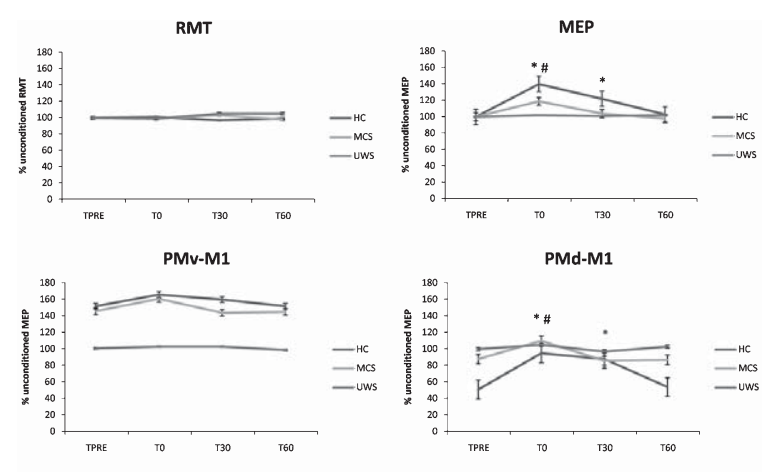

VEP latency
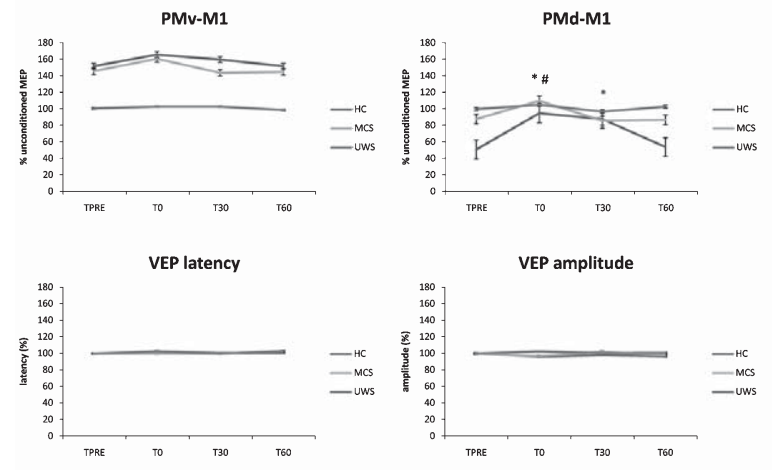

VEP amplitude

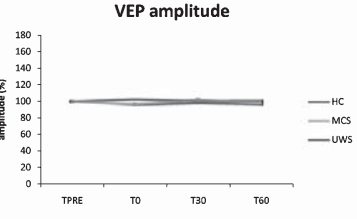

ERP-LO latency

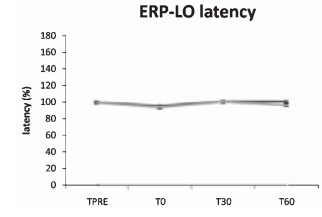

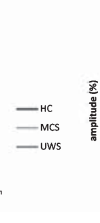

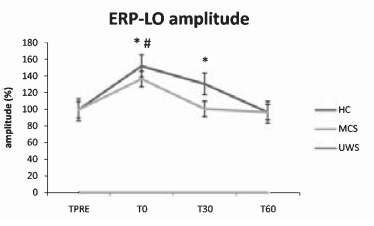

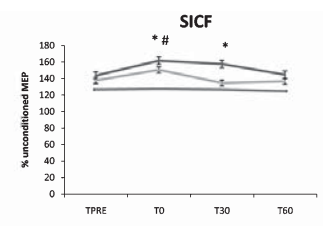
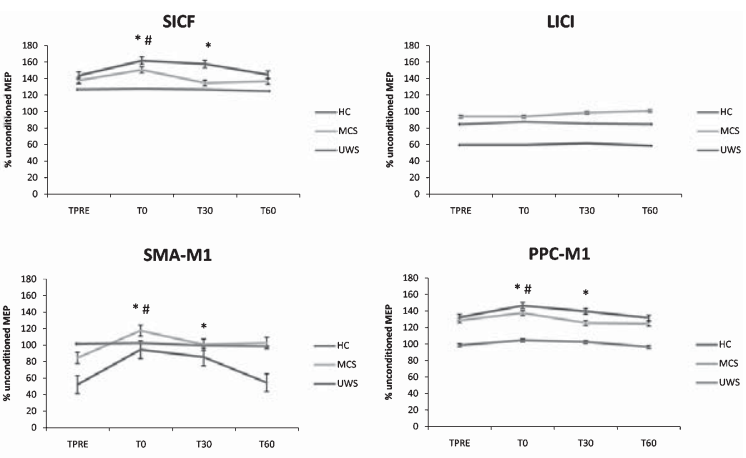

PPC-M1
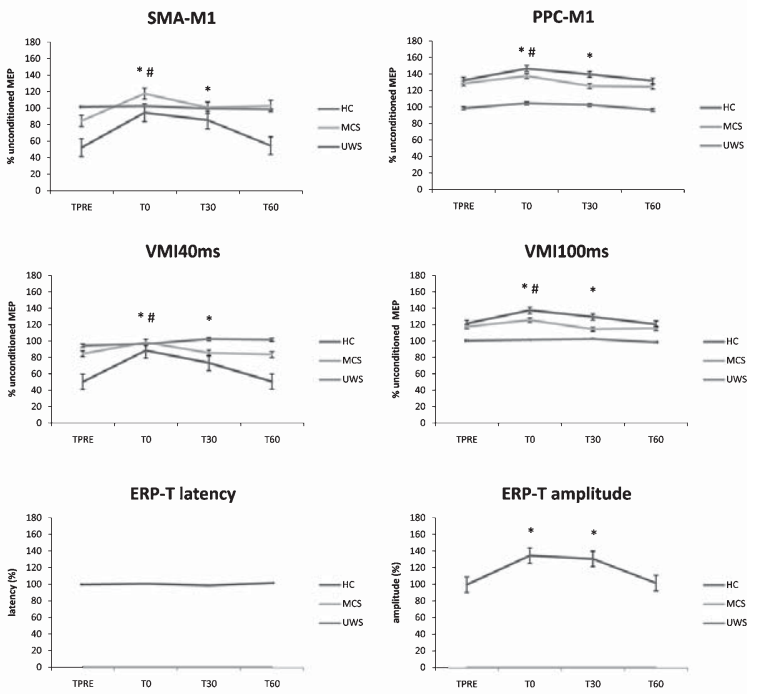

Fig. 2. The electrophysiological after-effects following the real_protocol (the ineffective protocols are not shown), which were significant up to $\mathrm{T}_{30}$ for $\mathrm{HC}^{(*)}$ ), and to $\mathrm{T}_{0}$ for MCS (\#). UWS patients did not show any significant after-effects (except for n. 3). Indeed, UWS showed before and after the real_protocol a wide impairment of cortical excitability and connectivity, whereas MCS showed an amelioration of such parameters. Values are expressed as percent of the unconditioned one. Error bars refer to standard error. 


\subsection{Conditioning protocol electrophysiological effects}

We summarized the statistical analysis data in Table 3. RMT, LICI, ERP $\mathrm{ELO}_{\text {and }} \mathrm{ERP}_{\mathrm{LT}}$ latency, PMv-M1, and VEP latency and amplitude did not significantly vary after each conditioning protocol. Concerning MEP, ERP LLO $_{\text {amplitude, PMd-M1, and }}$ SICF, we observed a significant amplitude increase at $\mathrm{T}_{0}$ and $\mathrm{T}_{30}$ in $\mathrm{HC}$ (the electrophysiological values reverted at $\mathrm{T}_{60}$ at the baseline ones), and in MCS at $\mathrm{T}_{0}$ (the electrophysiological values reverted at $\mathrm{T}_{30}$ and $\mathrm{T}_{60}$ at baseline ones), only after the real_protocol, whereas none of the patients with UWS, but one (n.3), showed such effects (Fig. 2). PPC-M1, VMI $40 \mathrm{~ms}$, and $\mathrm{VMI}_{100 \mathrm{~ms}}$ after-effects following real_protocol were particularly evident either in $\mathrm{HC}$ (up to $\mathrm{T}_{30}$ ) or in MCS (at $\mathrm{T}_{0}$ ), but not in UWS, with the exception of the patient n. 3. The tDCS_alone protocol induced aftereffects that were similar to real_protocol, although non-significant and limited to MEP amplitude increase and PPC-M1 facilitation. Moreover, there were no effects to be referred to visuomotor processes. Either the tACS_alone or the sham_protocol were totally ineffective. Figure 2 shows the percentual changes of the electrophysiological parameters. Concerning the UWS subject n. 3, we found MCS-like real_protocol aftereffects at $\mathrm{T}_{0}$, which were $\sim 14 \%$ lower in strength than the mean values of patients with MCS. Moreover, we identified a small ERP $P_{\text {LLO }}$ only at $\mathrm{T}_{0}$.

\subsection{Correlations}

We assessed the correlations between the amounts of electrophysiological $\mathrm{T}_{\mathrm{PRE}} / \mathrm{T}_{0}$ changes in $\mathrm{HC}$ group, in an attempt to better understand the direction of the effects induced by the real_protocol. Interestingly, the VMI\% increase was related to the PMd-M1\% $(\mathrm{Z}=3.5, p=0.001)$ and the SMA-M1\% increase $(Z=2, p=0.04)$. Moreover, we observed a correlation between the PPC-M1 facilitation and the $\mathrm{ERP}_{\mathrm{LT}}$-amplitude increase $(\mathrm{Z}=3, p=0.006)$.

\section{Discussion}

Recent neurophysiological and functional neuroimaging studies have suggested that the lack of behavioral responsiveness in patients affected by DOC does not necessarily imply unawareness, since a patient could not show purposeful behavior owing to motor-output or cognitive deterioration rather than connectivity impairment (Bruno et al., 2011; Formisano et al., 2013). Interestingly, some residual patterns of cortical connectivity within visuomotor areas have been previously shown in some individuals with UWS (Owen et al., 2006; Monti et al., 2010, 2013).

For the first time ever, we assessed the presence of residual large-scale visuomotor functional connectivity in a DOC sample by means of an electrophysiological approach consisting of paired tDCS and tACS. Only the real_protocol induced a potentiation of the M1 excitability (MEP amplitude and SICF increase), the premotor-motor connectivity (increased facilitatory and decreased inhibitory connections), and the visual basic cognitive process (ERP $_{\mathrm{LT}}$ increase

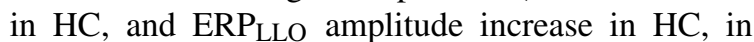
patients with MCS, and in one subject affected by UWS). In addition, such after-effects were strongly inter-correlated (VMI and PMd-M1, VMI and SMA-

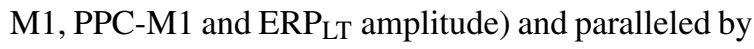
a transient visuomotor CRS-R score improvement in some patients (MCS n. 4 and 5; UWS n. 3). In detail, some patients moved from the "perceptual awareness" (i.e. the potential to perceive the external world and to interact with it, expressed at least by the visual startle at the CRS-R) to a transient "visual consciousness" (at least visual fixation at the CRS-R). Nevertheless, since both some patients with UWS and MCS showed a stable visual fixation at the CRS-R (a score of 2 at visuomotor domain), the visual fixation could not be per se considered as a marker of MCS, as also suggested by functional neuroimaging studies (e.g. Bruno et al., 2010). On the other hand, we may argue that even UWS could have a residual potential to interact with external word through VMI processes, as also suggested by other studies concerning different sensory modalities, including pain processing (de Tommaso et al., 2015). Notably, a "general visual consciousness" (the highest grade of the VMI process that also enroll other extra-visual circuitries) needs more functional and structured visuomotor networks (O'Regan and Noë, 2011), which were not assessed by our protocol.

Hence, the after-effects of our real_protocol may suggest the possibility of a visuomotor output facilitation leading to a higher conscious level, even in some patients affected by UWS. This finding may be due to a potentiation of the residual premotormotor, visuomotor, and visuo-cognitive functions, 
thus suggesting a diagnosis of fLIS. Indeed, the preserved connectivity and the covert awareness may subtend a covert partial consciousness even in some patients with UWS (i.e. fLIS), as previously hypothesized (Laureys et al., 2010; Giacino and Zasler, 1995; Bekinschtein and Manes, 2008; Monti et al., 2013; Perrin et al., 2006). Classic and total LIS are instead characterized by nearly-normal brain connectivity.

\section{1. tDCS-tACS physiology}

A recent VEP-rTMS protocol induced a LTP/LTDlike plasticity within visuomotor areas (Suppa et al., 2015), probably by means of a topographically specific spike-timing dependent plasticity (STDP) within M1. Moreover, several studies have shown that tDCS may also induce cortical polarity changes within M1 in a LTP-likewise (involving voltage-gated sodium channels and glutamatergic NMDA receptors) (Rizzo et al., 2014; Liebetanz et al., 2002; Nitsche et al., 2005; Ridding et al., 2000; Ridding and Uy, 2003; KaelinLang et al., 2002; Naro et al., 2015a). Thus, our paradigm could have induced a LTP-like potentiation by means of hetero-synaptic STDP mechanisms within M1 (involving visual -tACS- and motor -TMSinputs). The induction of a STDP mechanism is further corroborated by the lack of substantial effects following either the tDCS_alone, or the tACS_alone, or the sham_protocol. The after-effects we observed seem therefore to depend on the combination of the tDCS and tACS protocols.

Concerning the neural pathways supporting our after-effects, several studies have proposed that primary visual cortices could firstly elaborate the visuo-spatial information to generate visual perception and rapidonset response (in analogy to other sensory-motor integration processes) (Keliris et al., 2010; Leopold and Logothetis, 1996; Saron et al., 2001; Tokimura et al., 2000; Valeriani et al., 1999; Sowman et al., 2014). Then, visual information may flow through extra-striate, temporal, parieto-occipital, DLPFC, premotor, and subcortical regions (maybe cerebellum and basal ganglia), in order to generate spatial localization processing, oculo-motor control, visual attention, motor planning and execution, and visually-guided motor command (Kravitz et al., 2011). The activity within the frontal, temporal, and parieto-occipital areas may oversee the conscious contents of visual information, whereas premotor and motor areas may organize conscious motor planning (Dolce et al., 2011). Hence, we may hypothesize that our protocol enrolled both dorsal and ventral visual pathways (Goodale and Milner, 1992), mainly involving the PPC and SMA (that have an updating effect of on visual feedback-based, active motor planning, motor attention, and motor intentions) (Desmurget and Grafton, 2000; Jeannerod et al., 1994; Ikkai and Curtis, 2011). In addition, we cannot exclude a possible role of the contralateral homologous areas and transcallosal connections, since we limited our measurements to the left hemisphere (Koch et al., 2009; Suppa et al., 2015).

Notably, we have to acknowledge other issues concerning the physiological effects of our combined real_protocol: i) meta-plasticity phenomena could play an important role in supporting and regulating our after-effects. Indeed, the sequential application of two tDCS protocols may have involved either homeostatic or non-homeostatic meta-plasticity mechanisms (Bienenstock et al., 1982; Davis, 2006), suggesting the preservation of stabilizing plasticity phenomena even in individuals with UWS; ii) Suppa et al. (2015) applied a pattern-reversal visual stimulation protocol (paired to rTMS) in an attempt to activate the left temporal hemiretina and focally trigger brain networks. We instead stimulated the entire left retina, and a double tDCS protocol was applied. Hence, we triggered brain networks with lower topographic specificity than the VEP-rTMS protocol; iii) since RMT, VEP and MEP parameters were not different at baseline between MCS and UWS, and RMT and VEP did not vary after the conditioning protocols, we can exclude the possibility that baseline cortical excitability or VEP differences could have influenced the after-effects. Moreover, in reason of the blinded condition of participants concerning the different experimental sessions, we may exclude differences in the attentive level in the HC participants (Stefan et al., 2004).

\section{2. tDCS-tACS after-effects in patients with DOC}

The potentiation of the M1 excitability, the premotormotor connectivity, the visuo-cognitive function, and the visuomotor output in our patients may suggest the induction of VMI processes through time-locked neural activities that encompass premotor and parietooccipital networks (spreading from the posterior to the anterior cortical areas and vice versa) and other subcortical areas (maybe including thalamus and basal ganglia) (Monti et al., 2013; Suppa et al., 2015; Thiebaut de Schotten et al., 2014; Bruno et al., 2010; Petrides and 
Pandya, 2006; Di et al., 2014; Dum and Strick, 2005; O'Shea et al., 2007; Buch et al., 2010). Interestingly, it has been shown that visual pursuits depend on the activity of the mesiofrontal-precuneal cortex, which represents an important hub within the neural correlates of consciousness and is clearly impaired in patients suffering from severe chronic DOC (Bruno et al., 2010). The re-appearance or the enhancement of such visuomotor responses could therefore express a functional upgrading -although transient - of the residual corticocortical and the brainstem-thalamo-cortical networks supporting VMI processes (Dolce et al., 2011; Bruno etal., 2010; Dietal., 2014; Riganello and Sannita, 2009).

It has been hypothesized that plasticity and connectivity recovery in individuals suffering from DOC might depend on the modulation of post-ischemic LTP (Crepelet al., 1993; Di Filippo et al., 2008), the production of specific neurotrophins (e.g. BrainDerived Neurotrophic Factor; Kokaiaet al., 1998), and the regulation of excitatory/inhibitory dynamics within cortical and thalamo-cortical circuits (Dinget al., 2011). Thereby, it is conceivable that one or more of these mechanisms may have been triggered by the real_protocol, and could have favored the recruitment of silent or stunned cortico-cortical and cortico-subcortical connections, maybe involving the Schiff's mesocircuit model (Schiff, 2010).

Moreover, the bottom-up (stimulus salience) and top-down attention mechanisms (spatial and featuredriven attention, task goals) following the interaction between the ventral and the dorsal visual streams could have an important role in the clinical after-effects (Corbetta and Shulman, 2002; Currasco, 2011; Block, 2011; Pinto et al., 2013). Nevertheless, such issue needs to be further investigated by means of proper visuomotor tasks.

\subsection{Study limitations, conclusions and future perspectives}

In our opinion, our study proposes a promising approach in an attempt to identify residual patterns of VMI and large-scale fronto-parietal connectivity in patients affected by severe DOC, including UWS.

Noteworthy, the small sample size and the consequent mixed etiology represent a main limiting factor in our study. Nonetheless, it is difficult to study a large sample of patients with DOC, since the negative outcome of such patients is still unfortunately high.
However, our data further support the importance of patient's cooperation independent diagnostic approaches, aimed at assessing the consciousness level and differentiating real UWS from the patients that are clinically unable to express awareness signs (Formisano et al., 2013; Bruno et al., 2011; Schnakers et al., 2014; Naro et al., 2014). In addition, the possibility to identify such partially preserved cortico-cortical and cortico-subcortical networks in DOC may be useful in the selection of candidate patients for the deep brain stimulation (Schiff et al., 2007) or therapeutic and rehabilitative trials by means of non-invasive neurostimulation approaches.

\section{References}

Bäumer, T., Schippling, S., Kroeger, J., Zittel, S., Koch, G., Thomalla, G., Rothwell, J.C., Siebner, H.R., Orth, M., \& Münchau, A. (2009). Inhibitory and facilitatory connectivity from ventral premotor to primary motor cortex in healthy humans at rest-a bifocal TMS study. Clin Neurophysiol, 120(9), 1724-1731.

Bekinschtein, T., \& Manes, F. (2008). Evaluating brain function in patients with disorders of consciousness. Cleve Clin J Med, 75S2, 71-76.

Bienenstock, E.L., Cooper, L.N., \& Munro, P.W. (1982). Theory for the development of neuron selectivity, orientation specificity and binocular interaction in visual cortex. J Neurosci, 2(1), 32-48.

Bikson, M., Datta, A., Rahman, A., \& Scaturro, J. (2010). Electrode montages for $\mathrm{tDCS}$ and weak transcranial electrical stimulation, role of "return" electrode's position and size. Clin Neurophysiol, 121(12), 1976-1978.

Block N. (2011). Perceptual consciousness overflows cognitive access. Trends Cogn Sci, 15(12), 567-575.

Bruno, M.A., Laureys, S., \& Demertzi, A. (2013). Coma and disorders of consciousness. Hand Clin Neurol, 118, 205-213.

Bruno, M.A., Vanhaudenhuyse, A., Schnakers, C., Boly, M., Gosseries, O., Demertzi, A., Majerus, S., Moonen, G., Hustinx, R., \& Laureys, S. (2010). Visual fixation in the vegetative state, an observational case series PET study. BMC Neurol, 10,35 .

Bruno, M.A., Vanhaudenhuyse, A., Thibaut, A., Moonen, G., \& Laureys, S. (2011). From unresponsive wakefulness to minimally conscious PLUS and functional locked-in syndromes, recent advances in our understanding of disorders of consciousness. $J$ Neurol, 258(7), 1373-1784.

Buch, E.R., Mars, R.B., Boorman, E.D., \& Rushworth, M.F. (2010). A network centered on ventral premotor cortex exerts both facilitatory and inhibitory control over primary motor cortex during action reprogramming. J Neurosci, 30(4), 1395-1401.

Carrasco M. (2011). Visual attention: The past 25 years. Vis Res, 51(13), 1484-1525. 
Civardi, C., Cantello, R., Asselman, P., \& Rothwell, J.C. (2001). Transcranial magnetic stimulation can be used to test connections to primary motor areas from frontal and medial cortex in humans. Neuroimage, 14(6), 1444-1453.

Corbetta M., \& Shulman, G. (2002). Control of goal-directed and stimulus-driven attention in the brain. Nat Rev Neurosci, 3(3), 201-215.

Crepel, V., Hammond, C., Chinestra, P., Diabira, D., \& Ben-Ari, Y. (1993). A selective LTP of NMDA receptor-mediated currents induced by anoxia in CA1 hippocampal neurons. J Neurophysiol, 70(5), 2045-2055.

Davare, M., Lemon, R., \& Olivier, E. (2008). Selective modulation of interactions between ventral premotor cortex and primary motor cortex during precision grasping in humans. J Physiol, 586(Pt11), 2735-2742.

Davis, G.W. (2006). Homeostatic control of neural activity, from phenomenology to molecular design. Annu Rev Neurosci, 29, 307-323.

de Tommaso, M., Navarro, J., Lanzillotti, C., Ricci, K., Buonocunto, F., Livrea, P., \& Lancioni, G.E. (2015). Cortical responses to salient nociceptive and not nociceptive stimuli in vegetative and minimal conscious state. Front Hum Neurosci, 9, 17.

Desmurget, M., \& Grafton, S. (2000). Forward modeling allows feedback control for fast reaching movements. Trends Cogn Sci, 4(11), 423-431.

Di Filippo, M., Tozzi, A., Costa, C., Belcastro, V., Tantucci, M., Picconi, B., \& Calabresi, P. (2008). Plasticity and repair in the post-ischemic brain. Neuropharmacology, 55(3), 353-362.

Di, H., Nie1, Y., Hu, X., Tong, Y., Heine, L., Wannez, S., \& Huang, W. (2014). Assessment of visual fixation in vegetative and minimally conscious states. BMC Neurology, 14, 147.

Ding, M.C., Wang, Q., Lo, E.H., \& Stanley, G.B. (2011). Cortical excitation and inhibition following focal traumatic brain injury. J Neurosci, 31(40), 14085-14094.

Dolce, G., Lucca, L.F., Candelieri, A., Rogano, S., Pignolo, L., \& Sannita, W.G. (2011). Visual pursuit in the severe disorder of consciousness. J Neurotrauma, 28(7), 1149-1154.

Dum, R.P., \& Strick, P.L. (2005). Frontal lobe inputs to the digit representations of the motor areas on the lateral surface of the hemisphere. J Neurosci, 25(6), 1375-1386.

Formisano, R., D’Ippolito, M., \& Catani, S. (2013). Functional locked-in syndrome as recovery phase of vegetative state. Brain Inj, 27(11), 1332.

Gall, C., Fedorov, A.B., Ernst, L., Borrmann, A., \& Sabel, B.A. (2010). Repetitive transorbital alternating current stimulation in optic neuropathy. NeuroRehabilitation, 27(4), 335-341.

Gall, C., Sgorzaly, S., Schmidt, S., Brandt, S., Fedorov, A., \& Sabel, B.A. (2011). Noninvasive transorbital alternating current stimulation improves subjective visual functioning and visionrelated quality of life in optic neuropathy. Brain Stimul, 4(4), 175-188.

Garcia-Larrea, L., \& Peyron, R. (2013). Pain matrices and neuropathic pain matrices, A review. Pain, 154(S1), S29-S43.

Gerrard, P., Zafonte, R., \& Giacino, J.T. (2014). Coma recovery scale-revised, evidentiary support for hierarchical grading of level of consciousness. Arch Phys Med Rehabil, 95(12), 2335-2341.

Giacino, J., Ashwal, T., Childs, S., Cranford, N., Jennett, R., Katz, B., Kelly, D.I., Rosenberg, J.P., Whyte, J.H., Zafonte, J., \& Zasler, N.D. (2002). The minimally conscious state, definition and diagnostic criteria. Neurology, 58(3), 349-353.

Giacino, J.T., \& Zasler, N.D. (1995). Outcome after severe traumatic brain injury, coma vegetative state and the minimally responsive state. J Head Trauma Rehab, 10(1), 40-56.

Goodale, M.A., \& Milner, A.D. (1992). Separate visual pathways for perception and action. Trends Neurosci, 15(1), 20-25.

Grossberg, S. (2003). How does the cerebral cortex work? Development, learning, attention, and 3-D vision by laminar circuits of visual cortex. Behav Cogn Neurol Rev, 2(1), 47-76.

Haig, A.J., Katz, R.T., \& Sahgal, V. (1987). Mortality and complications of the locked-in syndrome. Arch Phys Med Rehabil, 68(1), 24-27.

Hildebrandt, H., Happe, S., Deutschmann, A., Basar-Eroglu, C., Eling, P., \& Brunhöber, J. (2007). Brain perfusion and VEP reactivity in occipital and parietal areas are associated to recovery from hypoxic vegetative state. J Neurol Sci, 260(1-2), 150-158.

Humphreys, G.W., Riddoch, M.J., \& Price, J. (1997). Top-down processes in object identification: Evidence from experimental psychology, neuropsychology and functional neuroanatomy. Phil Trans R Soc Lond B, 352(1358), 1275-1282.

Ikkai, A., \& Curtis, C.E. (2011). Common neural mechanisms supporting spatial working memory, attention and motor intention. Neuropsychologia, 49(6), 1428-1434.

Jeannerod, M., Decety, J., \& Michel, F. (1994). Impairment of grasping movements following a bilateral posterior parietal lesion. Neuropsychologia, 32(4), 369-380.

Kaelin-Lang, A., Luft, A.R., Sawaki, L., Burstein, A.H., Sohn, Y.H., \& Cohen, L.G. (2002). Modulation of human cortico-motor excitability by somatosensory input. $J$ Physiol, 540(Pt2), 623633.

Katsuki, F., \& Constantinidis, C. (2012). Early involvement of prefrontal cortex in visual bottom-up attention. Nat Neurosci, 15(8), 1160-1166.

Keliris, G.A., Logothetis, N.K., \& Tolias, A.S. (2010). The role of the primary visual cortex in perceptual suppression of salient visual stimuli. J Neurosci, 30(37), 12353-12365.

Koch, G., Fernandez Del Olmo, M., Cheeran, B., Ruge, D., Schippling, S., Caltagirone, C., \& Rothwell, J.C. (2007b). Focal stimulation of the posterior parietal cortex increases the excitability of the ipsilateral motor cortex. J Neurosci, 27(25), 6815-6822.

Koch, G., Franca, M., Mochizuki, H., Marconi, B., Caltagirone, C., \& Rothwell, J.C. (2007a). Interactions between pairs of transcranial magnetic stimuli over the human left dorsal premotor cortex differ from those seen in primary motor cortex. J Physiol, 578(Pt2), 551-562.

Koch, G., Ruge, D., Cheeran, B., Fernandez Del Olmo, M., Pecchioli, C., Marconi, B., Versace, V., Lo Gerfo, E., Torriero, S., Oliveri, M., Caltagirone, C., \& Rothwell, J.C. (2009). TMS activation 
of inter-hemispheric pathways between the posterior parietal cortex and the contralateral motor cortex. J Physiol, 587(Pt17), 4281-4292.

Kokaia, Z., Andsberg, G., Yan, Q., \& Lindvall, O. (1998). Rapid alterations of BDNF protein levels in the rat brain after focal ischemia: Evidence for increased synthesis and anterograde axonal transport. Exper Neurol, 154(2), 289-301.

Kotchoubey, B., Veser, S., Real, R., Herbert, C., Lang, S., \& Kübler, A. (2013). Towards a more precise neurophysiological assessment of cognitive functions in patients with disorders of consciousness. Restor Neurol Neurosci, 31(4), 473-485.

Kravitz, D.J., Saleem, K.S., Baker, C.I., \& Mishkin, M. (2011). A new neural framework for visuo-spatial processing. Nat Rev Neurosci, 12(4), 217-230.

Laureys, S., Celesia, G.G., Cohadon, F., Lavrijsen, J., León-Carrión, J., Sannita, W.G., Sazbon, L., Schmutzhard, E., von Wild, K.R., Zeman, A., Dolce, G. and the European Task Force on Disorders of Consciousness. (2010b). Unresponsive wakefulness syndrome: A new name for the vegetative state or apallic syndrome. BMC Med, 8,68 .

Laureys, S., Faymonville, M.E., Luxen, A., Lamy, M., Franck, G., \& Maquet, P. (2010b). Restoration of thalamocortical connectivity after recovery from persistent vegetative state. Lancet, 355(9217), 1790-1791.

Laureys, S., Pellas, F., Van Eeckhout, P., Ghorbel, S., Schnakers, C., Perrin, F., Berre, J., Faymonville, M.E., Pantke, K.H., Damas, F., Lamy, M., Moonen, G., \& Goldman, S. (2005). The lockedin syndrome: What is it like to be conscious but paralyzed and voiceless? Prog Brain Res, 150,495-511.

Leopold, D.A., \& Logothetis, N.K. (1996). Activity changes in early visual cortex reflect monkeys' percepts during binocular rivalry. Nature, 379(6565), 549-553.

Liebetanz, D., Nitsche, M.A., Tergau, F., \& Paulus, W. (2002). Pharmacological approach to the mechanisms of transcranial DC stimulation induced after-effects of human motor cortex excitability. Brain, 125(Pt10), 2238-2247.

Machado, S., Arias-Carrión, O., Sampaio, I., Bittencourt, J., Velasques, B., Teixeira, S., Nardi, A.E., Piedade, R., \& Ribeiro, P. (2014). Source imaging of P300 visual evoked potentials and cognitive functions in healthy subjects. Clin EEG Neurosci, 45(4), 262-268.

Miranda, P.C., Lomarev, M., \& Hallett, M. (2006). Modeling the current distribution during transcranial direct current stimulation. Clin Neurophysiol, 117(7), 1623-1629.

Monti, M.M., Laureys, S., \& Owen, A.M. (2010). The vegetative state. BMJ, 341, c3765.

Monti, M.M., Pickard, J.D., \& Owen, A.M. (2013). Visual cognition in disorders of consciousness, from V1 to top-down attention. Hum Brain Mapp, 34(6), 1245-1253.

Naro, A., Calabrò, R.S., Russo, M., Leo, A., Pollicino, P., Quartarone, A., \& Bramanti, P. (2015a). Can transcranial direct current stimulation be useful in differentiating unresponsive wakefulness syndrome from minimally conscious state patients? Restor Neurol Neurosci, 33(2), 159-176.

Naro, A., Leo, A., Russo, M., Quartarone, A., Bramanti, P., \& Calabrò, R.S. (2015b). Shaping thalamo-cortical plasticity, a marker of cortical pain integration in patients with post-anoxic unresponsive wakefulness syndrome? Brain Stimul, 8(1), 97-104.

Naro, A., Russo, M., Leo, A., Bramanti, P., Quartarone, A., \& Calabrò, R.S. (2014). A single session of repetitive transcranial magnetic stimulation over the dorsolateral prefrontal cortex in patients with unresponsive wakefulness syndrome, preliminary results. Neurorehabil Neural Repair, DOI: $10.1177 / 1545968314562114$

Nitsche, M.A., Liebetanz, D., Lang, N., Antal, A., Tergau, F., \& Paulus, W. (2003). Safety criteria for transcranial direct current stimulation (tDCS) in humans. Clin Neurophysiol, 114(11), 2220-2222.

Nitsche, M.A., Seeber, A., Frommann, K., Klein, C.C., Rochford, C., Nitsche, M.S., Fricke, K., Liebetanz, D., Lang, N., Antal, A., Paulus, W., \& Tergau, F. (2005). Modulating parameters of excitability during and after transcranial direct current stimulation of the human motor cortex. J Physiol, 568(Pt1), 291-303.

O'Shea, J., Sebastian, C., Boorman, E.D., Johansen-Berg, H., \& Rushworth, M.F. (2007). Functional specificity of human premotor-motor cortical interactions during action selection. EurJ Neurosci, 26(7), 2085-2095.

O'Regan, J.K., \& Noë, A. (2011). A sensorimotor account of vision and visual consciousness. Behav Brain Sci, 24(5), 939-1031.

Owen, A.M., Coleman, M.R., Boly, M., Davis, M.H., Laureys, S., \& Pickard, J.D. (2006). Detecting awareness in the vegetative state. Science, 313(5792), 1402.

Perrin, F., Schnakers, C., Schabus, M., Degueldre, C., Goldman, S., Brédart, S., Faymonville, M.E., Lamy, M., Moonen, G., Luxen, A., Maquet, P., \& Laureys, S. (2006). Brain response to one's own name in vegetative state, minimally conscious state and locked-in syndrome. Arch Neurol, 63(4), 562-569.

Petrides, M., \& Pandya, D.N. (2006). Efferent association pathways originating in the caudal prefrontal cortex in the macaque monkey. J Comp Neurol, 498(2), 227-251.

Pinto, Y., van der Leij, A.R., Sligte, I.G., Lamme, V.A.F., \& Scholte, H.S. (2013). Bottom-up and top-down attention are independent. $J$ Vis, 13(3), 16.

Ridding, M.C., \& Uy, J. (2003). Changes in motor cortical excitability induced by paired associative stimulation. Clin Neurophysiol, 114(8), 1437-1444.

Ridding, M.C., Brouwer, B., Miles, T.S., Pitcher, J.B., \& Thompson, P.D. (2000). Changes in muscle responses to stimulation of the motor cortex induced by peripheral nerve stimulation in human subjects. Exp Brain Res, 131(1), 135-143.

Riganello, F., \& Sannita, W.G. (2009). Residual brain processing in the vegetative state. J Psychophysiol, 23(1), 18-26.

Rioult-Pedotti, M.S., Friedman, D., \& Donoghue, J.P. (2000). Learning-induced LTP in neocortex. Science, 290(5491), 533-536.

Rizzo, V., Terranova, C., Crupi, D., Sant'Angelo, A., Girlanda, P., \& Quartarone, A. (2014). Increased transcranial direct current stimulation after effects during concurrent peripheral electrical nerve stimulation. Brain Stimul, 7(1), 113-121. 
Rossini, P.M., Barker, A.T., Berardelli, A., Caramia, M.D., Caruso, G., Cracco, R.Q., Dimitrijevid, M.R., Hallett, M., Katayama, Y., Liicking, C.H., Maertens de Noordhout, A.L., Marsden, C.D. Murray, N.M.F., Rothwell, J.C., Swash, M., \& Tomberg, C. (1994). Non-invasive electrical and magnetic stimulation of the brain, spinal cord and roots, basic principles and procedures for routine clinical application. Report of an IFCN committee. Electroencephalogr Clin Neurophysiol, 91(2), 79-92.

Sabel, B.A., Fedorov, A.B., Naue, N., Borrmann, A., Herrmann, C., \& Gall, C. (2011). Non-invasive alternating current stimulation improves vision in optic neuropathy. Restor Neurol Neurosci, 29(6), 493-505.

Sanes, J.N., \& Donoghue, J.P. (2000). Plasticity and primary motor cortex. Anпи Rev Neurosci, 23, 393-415.

Saron, C.D., Schroeder, C.E., Foxe, J.J., \& Vaughan, H.G. Jr. (2001). Visual activation of frontal cortex, segregation from occipital activity. Brain Res Cogn BrainRes, 12(1), 75-88.

Schiff, N.D. (2010). Recovery of consciousness after brain injury: A mesocircuit hypothesis. Trends Neurosci, 33(1), 1-9.

Schiff, N.D., Giacino, J.T., Kalmar, K., Victor, J.D., Baker, K., Gerber, M., Fritz, B., Eisenberg, B., Biondi, T., O'Connor, J., Kobylarz, E.J., Farris, S., Machado, A., McCagg, C., Plum, F., Fins, J.J., \& Rezai, A.R. (2007). Behavioral improvements with thalamic stimulation after severe traumatic brain injury. Nature, 448(7153), 600-603.

Schnakers, C., Giacino, J.T., Løvstad, M., Habbal, D., Boly, M., Di, H., Majerus, S., \& Laureys, S. (2014). Preserved Covert Cognition in Non-communicative Patients With Severe Brain Injury? Neurorehabil Neural Repair, 29(4), 308-317.

Sowman, P.F., Dueholm, S.S., Rasmussen, J.H., \& MrachaczKersting, N. (2014). Induction of plasticity in the human motor cortex by pairing an auditory stimulus with TMS. Front Hum Neurosci, 8, 398 .

Stefan, K., Kunesch, E., Cohen, L.G., Benecke, R., \& Classen, J. (2000). Induction of plasticity in the human motor cortex by paired associative stimulation. Brain, 123(Pt3), 572-584.

Stefan, K., Wycislo, M., \& Classen, J. (2004). Modulation of associative human motor cortical plasticity by attention. $\mathrm{J} \mathrm{Neu}$ rophysiol, 92(1), 66-72.

Suppa, A., Biasiotta, A., Belvisi, D., Marsili, L., La Cesa, S., Truini, A., Cruccu, G., \& Berardelli, A. (2013). Heat-evoked experimental pain induces long-term potentiation-like plasticity in human primary motor cortex. Cereb Cortex, 23(8), 1942-1951.
Suppa, A., Li Voti, P., Rocchi, L., Papazachariadis, O., \& Berardelli, A. (2015). Early Visuo-motor Integration Processes Induce LTP/LTD-Like Plasticity in the Human Motor Cortex. Cereb Cortex, 25(3), 703-712.

The Multi-Society Task Force on PVS. (1994). Medical aspects of the persistent vegetative state. $N$ Engl J Med, 330(21), 1499-1508.

Thiebaut de Schotten, M., Urbanski, M., Valabregue, R., Bayle, D.J., \& Volle, E. (2014). Subdivision of the occipital lobes, an anatomical and functional MRI connectivity study. Cortex, $56,121-137$.

Tokimura, H., Di Lazzaro, V., Tokimura, Y., Oliviero, A., Profice, P., Inolsa, A., Mazzone, P., Tonali, P., \& Rothwell, J.C. (2000). Short latency inhibition of human hand motor cortex by somatosensory input from the hand. $J$ Physiol, 523(Pt2), 503-513.

Tokimura, H., Ridding, M.C., Tokimura, Y., Amassian, V.E., \& Rothwell, J.C. (1996). Short latency facilitation between pairs of threshold magnetic stimuli applied to human motor cortex. Electroencephalogr Clin Neurophysiol, 101(4), 263-272.

Trojano, L., Moretta, P., Loreto, V., Cozzolino, A., Santoro, L., \& Estraneo, A. (2012). Quantitative assessment of visual behavior in disorders of consciousness. J Neurol, 259(9), 1888-95.

Valeriani, M., Restuccia, D., Di Lazzaro, V., Oliviero, A., Profice, P., Le Pera, D., Saturno, E., \& Tonali, P. (1999). Inhibition of the human primary motor area by painful heat stimulation of the skin. Clin Neurophysiol, 110(8), 1475-1480.

Valls-Sole, J., Pascual-Leone, A., Wassermann, E.M., \& Hallett, M. (1992). Human motor evoked responses to paired transcranial magnetic stimuli. Electroencephalogr Clin Neurophysiol, 85(6), 355-364.

Wolters, A., Sandbrink, F., Schlottmann, A., Kunesch, E., Stefan, K. Cohen, L.G., Benecke, R., \& Classen, J. (2003). A temporally asymmetric Hebbian rule governing plasticity in the human motor cortex. J Neurophysiol, 89(5), 2339-2345.

Ziemann, U., Paulus, W., Nitsche, M.A., Pascual-Leone, A., Byblow, W.D., Berardelli, A., Siebner, H.R., Classen, J., Cohen, L.G., \& Rothwell, J.C. (2008). Consensus, motor cortex plasticity protocols. Brain Stimul, 1(3), 164-182. 